\title{
El razonamiento sofístico (privado) y la teoría de los predicables
}

\author{
José Miguel Gambra \\ Universidad Complutense
}

\begin{abstract}
The aristotelian doctrine of fallacies in the Sophistical Refutations cannot be understood without reference to Topics. In this paper we attempt to show that, in this last work, there is an implicit "logic of predicables", which can explain in a very convincing way some of those fallacies, like those of accident, of consequent, secundum quid ad simpliciter and babbling. At the same time, we present the main ingredients of this logic and we discuss some modern solutions about those sophisms.
\end{abstract}

\section{Introducción}

En lógica, como en tantas otras ciencias, la autoridad de Aristóteles ha sido de enorme trascendencia y duración. Sin embargo, algunas de sus doctrinas han sido tan manoseadas, han pasado por tantas interpretaciones, que casi no se las reconoce. Algunos de los ejemplos más notables de este fenómeno se hallan en la teoria de las falacias o sofismas.

Los manuales anglosajones acostumbran a introducir un capitulo dedicado a las falacias, cosa no tan común entre los nuestros. Allí aparecen por lo general los trece sofismas que expone Aristóteles en los primeros capitulos de Las Refutaciones Sofisticas. Pero lo que queda de ellos no es, a menudo, más que el nombre. Uno de los casos más llamativos es el sofisma del accidente. Asi Copi, en su conocidisima Introducción a la Lógica (Buenos Aires: Eudeba 1974, p. 92), llama de esta manera la falacia "que consiste en aplicar una 
regla general a un caso cuyas circunstancias 'accidentales' hacen inaplicable la regla"l. Cometería esta falacia quien devolviera unas armas prestadas a alguien que se ha vuelto loco por aplicar la norma general que obliga a devolver lo prestado.

Si tornamos la mirada a lo que Aristóteles llama sofisma del accidente ( $\pi \alpha \varrho \alpha$ $\tau \delta$. $\sigma \nu \mu \beta \varepsilon \beta \eta x o ̀ \varsigma ~ \pi \alpha \lambda \sigma \gamma l \sigma \mu \delta \zeta)$ nos encontraremos, entre otros, con estos ejemplos (que he modificado ligeramente para mayor claridad):
A) Corisco es el que viene
El que viene es un desconocido para ti
Luego, Corisco es un desconocido para ti
(Ref. Sof. 24, 179a 34)
B) Este perro es padre
Este perro es tuyo
Luego este perro es tu padre
(Ref. Sof. 24, 179a 35)

Ya a primera vista se puede advertir que estos razonamientos incorrectos nada tienen que ver con el de Copi. Pero si consultamos alguno de los párrafos donde Aristóteles explica el error que en ellos se comete, esto será todavía más claro.

He aquí alguno de esos textos:

I. "En los paralogismos debidos à accidente, el error procede de la incapacidad de discernir lo que es idéntico de lo que es otro, y de lo que es uno de lo que es múltiple, o para qué clases de predicados todos los accidentes son los mismos que para el sujeto" (Ref. Sof. 7, 169b 3-6).

1 W. S. Jevons ofrece la misma interpretación del sofisma de accidente. Cfr. Lógica, Pegaso 1941, p. 167. 
II. "Es evidente que, en todos los casos no es necesario que lo que es verdadero del accidente lo sea también de la cosa. Pues sólo a las cosas que son indiferenciadas en su esencia y que son una y la misma cosa parece que pertenecen las mismas cosas" (Ref. Sof. 24, 179a 34).

Dejamos para luego el análisis de estos textos, que - falta hace reconocerlo- no son muy esclarecedores para captar, a primera vista, cuál es para Aristóteles el error cometido en estos sofismas. También resulta obscuro, dada la gran diferencia en su estructura, qué hay de común en los sofismas mencionados para aunarlos bajo una sola denominación.

La historia ha puesto de manifiesto cuán obscuros y breves son estos párrafos. En efecto, de los comentaristas griegos y latinos que se han ocupado de las Refutaciones sofisticas no he hallado ninguno que resuelva de manera satisfactoria esta cuestión.

En los textos citados, la noción más importante es, sin duda, la de accidente, que da nombre a estos sofismas. Pues bien muchos comentaristas, desde Alejandro de Afrodisia hasta Ockham ${ }^{2}$, entendieron que Aristóteles usa aquí la palabra accidente con el sentido genérico de predicado cualquiera, y no con alguno de los sentidos, más precisos, a modo de predicación accidental (accidente predicable) o modo de ser accidental (accidente predicamental o categorial). De ahí que la frase clave "no es necesario que lo que se dice del accidente se diga también de la cosa" haya sido entendida como si dijera "no hay necesidad de que si un atributo se dice de algo y esto se dice de otra cosa entonces aquel atributo se diga de esta cosa". De esta manera resulta que comete la falacia del accidente cualquier tipo de silogismo que no se atenga a los modos válidos. Por este camino, durante la edad media, se desdibujó completamente el sentido de nuestro sofisma y esta denominación acabó, como hemos visto por aplicarse a incorrecciones que no

2 Cfr. J. M. Gambra: "Medieval Solutions to the Sophism of Accident", en K JACOBI (ed.): Argumentationstheorie, Leiden: Brill 1993. 
tenían la más remota relación con los ejemplos y explicaciones del estagirita.

El sofisma de Corisco y otros similares constituyen, sin embargo, una verdadera dificultad lógica, con caracteres muy definidos. De ahí que, andando el tiempo, reaparezca este mismo problema en la historia de la lógica, aunque ya sin la denominación de sofisma del accidente.

Otra de las palabras claves en los textos de Aristóteles es la de identidad (lo indiferenciado, lo que es uno y lo mismo). Pues bien, precisamente al estudiar las leyes de la identidad, los lógicos modernos acabaron por topar con los mismos sofismas de Aristóteles. Entre estas leyes, en efecto, es quizás la más importante la de intercambio de idénticos que dice: "si dos nombres denotan la misma cosa singular (es decir son idénticos), entonces puede substituirse uno por otro en cualquier proposición". Ahora bien, hay ejemplos de razonamientos que parecen cumplir esta regla y que, sin embargo, son evidentemente inválidos. El más famoso de ellos es el siguiente:

Jorge IV quiso saber si Scott era el autor de Waverley

Scott = el autor de Waverley

Luego Jorge IV quiso saber si Scott era Scott.

(Cfr. B. RuSsEll: "On denoting", Mind XIV (1905), pp. 479-493).

Las premisas pueden ser verdaderas, pero la conclusión es evidentemente falsa, pues; aunque hubiera sido muy romo - que no lo fue- el entendimiento de ese monarca, no parece posible que su curiosidad se dirigiera a cuestiones tan simples.

Esta falacia es la misma que se comete en el ejemplo de Corisco ${ }^{3}$. Para verlo basta con señalar que la proposición "Corisco es el que

3 BETH incluye alguno de los ejemplos aristotélicos de sofisma entre las paradojas del análisis y la denotación (Foundations of Mathematics, Amsterdam: NorthHolland 1959, p. 179). 
viene" es una identidad semejante a la segunda proposición de la falacia de Scott.

Las soluciones que los lógicos contemporáneos han buscado para las paradojas de la denotación y del análisis son francamente insatisfactorias y por lo general circulares. Consisten, en poner límites al intercambio de idénticos, diciendo por ejemplo, que esa regla no vale en contextos intensionales (R. CARNAP, Meaning and Necessity, Chicago: The University of Chicago Press 1956, cap. IIII) o en contextos de referencia opaca (W. V. O. QUINE: Desde un Punto de Vista Lógico, Orbis 1984, cap. VIII). Pertenecen a tales contextos, entre otras, las oraciones donde aparecen términos epistémicos (conocer y otros por el estilo) o términos entrecomillados. Pero si queremos saber cómo se distinguen estos contextos de aquellos donde vale la regla de intercambio, se responde que pertenecen a esa clase de contextos aquellas oraciones en las que no vale la regla de intercambio, con lo cual se cae, como dije, en la circularidad o en la explicación meramente ad hoc.

\section{La lógica de los predicables}

Un sofisma es un discurso que parece seguir las reglas de un cálculo, pero al que le falta algo para cumplir las reglas del cálculo de que se trate. Así, concluir de una implicación y de la afirmación del consecuente la afirmación del antecedente, es un sofisma, porque parece seguir las reglas de la lógica de enunciados ${ }^{4}$ pero no las sigue. Los sofismas son tales por relación a unas leyes lógicas que parecen seguir. Los medievales decían que para definir adecuadamente un sofisma hacia falta determinar la causa de la apariencia (tener una estructura similar a una ley válida) y la causa de la no existencia (incumplir alguna de las exigencias de esa ley).

\footnotetext{
4 Parece que cumple el modus ponens de la implicación, pero se confunde el antecedente con el consecuente; también puede decirse que parece cumplir el modus ponens de la equivalencia, pero se comete el error de no distinguir entre la implicación y la equivalencia. Ya que un sofisma puede parecer que cumple varias reglas de un mismo cálculo, sin acomodarse a ninguna de ellas, digo que un sofisma es tal por relación a un cálculo y no por relación a una regla particular.
} 
Si lográramos descubrir la clase de reglas que, a juicio de Aristóteles, parece seguir, pero no sigue, el sofisma del accidente, comprenderiamos el sentido que para él tenía esta falacia y otras que luego veremos. Creo haber encontrado esas reglas o leyes en lo que se puede llamar lógica de los predicables. Para presentar esta lógica empezaré por establecer una lista de signos que emplearé para simbolizar sus leyes.

Signos de cosas singulares: a,b,c...

Signos de universales: $A, B, C .$.

Signo del accidente exclusivo: ;

Superindices: ${ }^{\mathrm{e}}, \mathrm{g}$, di de, $\mathrm{p}$, , n .

Signo de identidad: $=$

Funtores de la lógica de enunciados y paréntesis.

Los signos de universales expresarán términos generales de cualquier categoría. Los signos de cosas singulares designarán individuos de cualquier categoría como "este blanco"s o "Corisco". Aristóteles atiende además a una forma de designar a los individuos que merecè especial atención: me refiero al caso en que un objeto singular se designe por medio de un accidente que sólo a él corresponda, como ocurre cuando para hablar de Sócrates lo denominamos como "el que está sentado". En tal caso se emplea un universal para hablar de un individuo, cosa que simbolizaré por medio del signo que denota al universal de que se trate, precedido de lo que he llamado "signo del accidente exclusivo". Por tanto, si $A$ significa "sentado", "el que está sentado" se simbolizará como $i A^{6}$.

5 Por "este blanco" entiendo un color singular que sometemos a nuestra consideración en unas circunstancias determinadas, como, por ejemplo, cuando decimos que este blanco es mate o que este blanco está o inhiere en esta tiza. Para

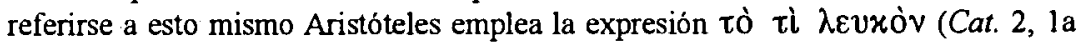
27).

${ }^{6}$ Aristóteles dice que, en tales casos, se emplean los accidentes como "propiedades en algunas ocasiones" y pone el ejemplo siguiente: "el que está sentado, que es un accidente, cuando un solo individuo esté sentado, será un propio en esa ocasión" (Top. I, 5, 102b 23, Cfr: Top. I, 7, 103a 32 ss.). 
En toda proposición, según Aristóteles, se afirma algo de algo ( $D e$ Int. 10, 19b 5). Lo que se dice de algo es siempre universal, pues los singulares no pueden predicarse $\left(A n \operatorname{Pr}\right.$. I, 27, 43a 39) ${ }^{7}$. En cambio, aquello de lo que se predica, el sujeto, puede ser universal o singular (An Pr. I, 27, 43a 39). Para representar las proposiciones que así pueden formarse, recurriremos a fórmulas con la estructura de funciones monádicas, donde el signo del predicado se pone a la izquierda y el del sujeto a la derecha. Entre ambos situaremos, cuando sea posible ${ }^{8}$, uno de los superíndices que expresan los modos de predicación, como pronto veremos. El predicado o el sujeto, cuando son universales, pueden estar compuestos de otros predicados, cosa que acaece, por ejemplo, en las definiciones. $\mathrm{Al}$ simbolizar semejantes predicados haremos uso de paréntesis de modo que se aúnen los signos de universales que se componen. Cuando el sujeto y el predicado sean idénticos por el número -de lo cual hablaremos en breve- se colocará entre las letras el signo de la identidad, seguido, siempre que se conozca, del superíndice correspondiente al tipo de identidad de que se trate.

En las primeras obras del Organon, Aristóteles distingue varias maneras de decirse el predicado del sujeto. Estos modos de predicación o predicables son el elemento más característico de la lógica que ahora estudiamos. Veamos brevemente una descripción de los mismos, acompañada de un ejemplo y su correspondiente representación simbólica9:

7 Cuando lo que se dice de un sujeto es un accidente exclusivo o usado como propiedad, parece que nos hallamos, según Aristóteles, ante un caso especial de predicación accidental donde, además, como luego veremos, hay identidad entre los términos de la proposición.

8 Cuando los textos de Aristóteles no permitan determinar claramente cuál es el modo de una predicación, se omitirá el superíndice entre los signos del sujeto y del predicado.

${ }^{9}$ Esta exposición parte de una idea que aquí se da por supuesta sin explicaciones: la noción de esencia o quididad de las cosas singulares. 
a) Especie es el predicado simple, que sólo se dice de individuos ${ }^{10}$, de tal manera que expresa con una sola palabra la esencia o quididad de esa cosa: "Corisco es hombre" $\rightarrow A^{e} b$.

Dada una especie ínfima, veámos ahora los predicados que de ella pueden decirse, pues la especie es la noción en torno a la cual se articulan todos los modos de predicación ${ }^{11}$.

b) Género es el predicado que expresa la esencia, pero sólo en aquella parte que es común a otras especies (Top. IV, 6, 144a 24): "El hombre es animal" $\rightarrow A^{g} B$.

c) Diferencia es el predicado que expresa la esencia del sujeto, pero sólo en la parte que es característica y exclusiva de la especie ${ }^{12}$ : "El hombre es bípedo" $\rightarrow A^{d i} B$.

Género y diferencia (tal como son presentados en los Tópicos) son más amplios que el sujeto, y no son, por tanto, intercambiables con el sujeto.

d) Definición es el predicado compuesto de género y diferencia que expresa completamente la esencia del sujeto. La definición, por

10 Cfr. An.. Post.. II, 13, 96b 5 ss.. Vid. J. M. Gambra: "La Lógica Aristotélica de los Predicables", Anuario Filosófico XXI/2 (1988), pp. 89-118, nota 5. Aquí por especie sólo se entenderá la especie átoma o última, no las especies intermedias, que son géneros respecto de otras inferiores.

11 La especie no es incluida por Aristóteles en la lista de los predicables que aparece en los Tópicos $(I, 5)$, seguramente porque los demás modos de predicación se definen tomando como sujeto de predicación una especie infima (Cfr. D. Ross, Aristotle, Londres: Methuen 1971, p. 57).

12 Aristóteles no incluye la diferencia en su primera enumeración de los predicables (Top. I ,5), aunque posteriormente la usa como tal en los Tópicos. Ross observa que la noción de diferencia es usada en esta obra y en los Segundos Analiticos (II, 13, 96a 24-96b 14) de manera diversa a como aparece en la Metafisica (II, 7). En aquellas obras la diferencia parece ser un universal más amplio que la especie, pero que unido al género constituye la definición de la especie. En la Metafisica en cambio, la diferencia es el universal que expresa la nota característica y exclusiva de la especie, de modo que no caben dos especies, aunque sean de géneros distintos, que incluyan en su definición las misma diferencia. 
tanto es intercambiable con la especie (Top. VI, 6, 143b 7; Met. VII, $11,1037 b$ 28): "El hombre es animal bipedo" $\rightarrow(A B)^{d e} C^{13}$.

e) Propiedad es el predicado que no expresa la esencia (en este sentido es accidental, Met. V, 30, 1025a 29) aunque sí está conectado con ella, hasta el punto de que sólo puede decirse de los miembros de la especie y de todos ellos. La propiedad es, pues intercambiable en la predicación (Top. I, 5, 102a 18) ${ }^{14}$ : "El hombre es risible" $\rightarrow$ $A^{p} B$.

f) Accidente es el predicado que se dice con verdad del sujeto, pero sin ninguna relación con la esencia del sujeto (no es ninguno de los otros predicados). Por ello mismo, también lo define Aristóteles como lo que puede darse o no darse en el sujeto, dado que todos los otros predicados, al hallarse conectados con la esencia, son necesarios (Cat. 5, 2a 14 y Top. I, 5, $102 \mathrm{~b} 3$ ss.) $)^{15}$ : "El hombre es blanco" $\rightarrow A^{a} B$.

Como hemos visto, todos estos modos de predicación pueden darse en una proposición con sujeto universal, pero también con

${ }^{13}$ Frecuentemente usaremos, para simbolizar el definiens, un predicado simple de la forma siguiente: $A^{d e} B$. Estrictamente hablando deberiamos, sin embargo, utilizar siempre un predicado complejo.

${ }^{14}$ El nexo que existe entre la especie y la propiedad parece que es el siguiente: son propiedades de una especie aquellos atributos universales tales que no forman parte de la definición de la especie, pero de cuya definición forma parte la especie ( $A n$ Post. I, 4, 73a 37 y I, 22, 84a 12).

15 Debe distinguirse el àccidente predicable, que es un modo de predicación, del accidente predicamental, es decir, de las cosas que están bajo una de las categorias accidentales. Entre ambos se dan estas dos diferencias de carácter lógico: 1) accidente predicamental pueden serlo tanto cosas singulares como universales, mientras que sólo lo universal es accidente predicable; 2) los accidentes predicamentales lo son con entera independencia de la predicación, mientras que los accidentes predicables sólo lo son por relación a un sujeto. Esto explica que un universal que pertenezca a una categoría accidental (v.gr. color) pueda decirse de un sujeto conforme a un modo esencial de predicación (v.gr. este blanco). Cabe incluso que algo perteneciente a una categoria no accidental se diga accidentalmente de un sujeto accidental (Met. V, 7, 1017a 13 ss.). 
sujeto singular, aunque siempre por relación a la especie del sujeto. (Habremos de añadir a las casos anteriores $A^{d i} b$ etc.).

Entre sujeto y predicado puede darse, además de la relación de predicación, la de identidad. La identidad, junto con la igualdad y la semejanza, son relaciones que tienen por fundamento la unidad. Conviene, pues, dedicar alguna atención a la idea de unidad que Aristóteles estudia en la Metafisica, en lo que, a este respecto, parece convenir con los Tópicos.

La unidad no es una noción relativa: no se dice de algo que es "uno" en orden a otra cosa, sino que, tal como la emplea Aristóteles, puede decirse sin más de un sujeto en singular, lo cual no impide que, en ocasiones, pueda también decirse de dos cosas que son una (en singular).

Aristóteles distingue entre la unidad o indivisibilidad ${ }^{16}$ por la esencia $(\lambda \sigma \gamma \circ \varsigma)$ y la unidad o indivisibilidad por el número (Met. $X, 1,1052 a$ ss.). Es uno por el número aquello cuya materia es una, es decir, lo que es individual (Met. X,1, 1052a 30 ss y Met. V, 7, $1016 \mathrm{~b} 33)$, puesto que la materia es el principio de individuación. Es uno por la esencia aquello cuya esencia es una o, por relación al conocimiento, aquello cuya intelección es una (Met. X, 1, 1052a 30 ss.). No tiene sentido decir de dos individuos que son numéricamente uno (en singular). Cabe, por el contrario, decir de dos individuos que son esencialmente uno, cuando ambos tiene la misma esencia o intelección. Esto último puede suceder bien porque la esencia completa es una "y entonces se da la unidad por la especie-, bien cuando lo que es uno es la parte de la esencia común a varias especies, y entonces la unidad es genérica (Met. V, 6, 1016b 34). Cuando algo es indivisible numéricamente, lo es también por la especie (pues lo que es individual tiene una sola forma especifica), pero no a la inversa. A su vez, lo que es uno especificamente lo es también por el género, pero no a la inversa (Met. V, 6, 1016b 35).

16 "El ser de lo uno es el ser de lo indivisible" (Met. X, 1, 1052b 16). 
La identidad es, según vimos, una relación. Por ello Aristóteles emplea las nociones de "idéntico", y sus opuestos "otro" y "diferente" como términos relativos, que se dicen de un sujeto en plural y, cuando se dicen de un sujeto singular, se hace por orden a otro.

La identidad por $\mathrm{si}^{17}$ se divide de tantas maneras como hemos dividido la unidad, pues aquélla se funda en ésta. Esto da lugar a una partición de la identidad, cuya más clara exposición se halla en los Tópicos $^{18}$.

Lo idéntico, en Tópicos I, 7, se subdivide primero en identidad por el género, la especie y el número. Son idénticas por el género las cosas que, siendo individualmente varias, se hallan, sin embargo, bajo el mismo género, como este caballo y este hombre. Son especificamente idénticas las cosas numérica $o$ individualmente diversas que, no obstante, pertenecen a la misma especie, como Sócrates y Corisco (Top. I, 7, 103a 10 ss.). Identidad específica y genérica tienen por fundamento la unidad que hemos llamado formal, bien por la especie, bien por el género. Los idénticos por el número son los que tienen una unidad numérica o material. En este caso no hay dos individuos sino sólo uno. ¿Cómo es posible que se dé una relación como la identidad cuando sólo hay una cosa? La respuesta es apuntada por Aristóteles en la Metafisica cuando dice que la identidad puede darse entre lo que, siendo uno, se toma como varios (Met. V, 9, 1018a 8), pero allí no se dice cómo puede suceder esto. La explicación se halla en los Tópicos, donde dice que "son idénticas en número las cosas en las que los nombres son múltiples, la cosa en cambio única" (Top. I, 7,103a 8).

17 Esta identidad se distingue de la identidad por accidente (Met. V, 9, 1017b 27), que aqui no vamos a considerar. Conviene, en todo caso no confundirla con la identidad numérica por accidente, de la cual luego hablaremos.

18 En la Metafisica, Aristóteles afirma que la identidad se apoya en la unidad. También apunta la primera subdivisión de la identidad, que seguidamente veremos, al hablar de las nociones de "lo otro", opuesta de forma general a la noción de lo idéntico (Met. V, 9, 1018a 10) y "diferente", que es una especie de lo que es "otro" (Met. V, 9, 1018a 12). No se ocupa, sin embargo, de la división de la identidad numérica. 
Entre los idénticos hay siempre, como hemos visto, cierta unidad. Sin embargo, al ser la identidad una relación, hace falta que haya algún tipo de diversidad o pluralidad, pues los términos de la relación son dos cuando menos. La identidad específica conlleva unidad esencial, pero no material, de modo que permite la diversidad de individuos. La identidad genérica implica unidad parcial en la esencia pero permite la pluralidad de especies y de individuos. Por el contrario la identidad numérica no permite ninguna clase de diversidad real, pues semejante unidad involucra la unidad esencial y material. Por tanto, si cabe una identidad fundada en la unidad numérica, esto es, una identidad numérica, tiene que haber una diversidad no fundada en los constituyentes reales de la cosa. Tal diversidad es la que se produce cuando llamamos a las mismas cosas con nombres diversos, lo cual puede producirse bien porque el concepto ( $\lambda$ ó $\gamma \circ \zeta)$ significado por tales nombres es diverso ${ }^{19}$, bien sencillamente por una casualidad lingüistica, en virtud de la cual hay nombres que significan lo mismo según la misma razón. En resumen, la identidad numérica se da entre una cosa en cuanto es denominada por un nombre y esa misma cosa en cuanto es denominada por otro nombre, siendo el concepto conforme a tales nombres bien el mismo bien diverso.

De aquí nace una subdivisión de la identidad numérica que, quizás por apoyarse directamente en los nombres y en el concepto a través del cual significan, no viene expuesta en la Metafisica sino en los Tópicos:

Cuando la diversidad entre los términos que denominan las mismas cosas es exclusivamente nominal, es decir, cuando las cosas designadas son las mismas y no es diferente la razón por la que tal denominación se da, entonces se produce la identidad numérica por el nombre. El ejemplo preferido de Aristóteles es el de "abrigo" y

19 Aristóteles, al tratar de los homónimos y sinónimos, admite que las cosas tienen un concepto conforme al nombre: así son sinónimas las cosas que tienen un nombre en común y el concepto conforme al nombre es el mismo (Cat. I, la 6). Creo que esta idea es muy útil para entender la identidad numérica. 
"gabán”. La predicación entre estos términos se expresará por medio de fórmulas como $A={ }^{n} B$.

Cuando las cosas son las mismas y el concepto por el que significan sus nombres es la esencia de esas cosas, pero en un caso se trata del nombre de la especie y en el otro de la definición de esa especie, tendremos dos nombres -especie y definición- que son idénticos numéricamente, pero por la definición. Verbigracia hombre y animal bípedo. El hombre es animal bípedo se simboliza por medio de $(A B)={ }^{d e} C$.

$\mathrm{Si}$, en tercer lugar, las cosas denominadas son las mismas, pero por un lado tenemos el nombre de la especie y, por otro, el nombre de una propiedad de esa especie, comoquiera que la propiedad en sentido estricto se dice de todos y de sólo los individuos de la especie, resultará que propiedad y especie son numéricamente idénticos por la propiedad. De esta manera son numéricamente idénticos hombre y risible. $A={ }^{p} B$ expresa que el hombre es risible.

Finalmente si, refiriéndonos a la misma cosa individual, de una parte la llamamos por su nombre propio y, de otra, por medio de un accidente exclusivo, el nombre propio y dicho accidente serán idénticos por el accidente (Top. I, 7, 103a 30 ss.). Por ejemplo Sócrates y el que está sentado son idénticos accidentalmente. También se puede, a mi juicio, incluir en este tipo de identidad la que se da entre dos accidentes exclusivos de la misma cosa singular. V.gr. el maestro de Platón y el que está sentado. Que Platón es el que está sentado se expresa por medio de $i A={ }^{a} b$. Que el maestro de Platón es el que está sentado se expresará por medio de $i A={ }^{a} i B$.

En el texto II de las Refutaciones antes citado arriba, y en otros lugares que luego consideraremos, Aristóteles habla de la identidad numérica por la esencia. ¿Cuáles de los modos vistos de identidad numérica es identidad por la esencia? Hay, sin duda, unidad o indiferenciación por la esencia entre las cosas que son idénticas por la definición (Met. X, 1, 1052b 16). Asimismo resulta innegable que entre las cosas que sólo por el nombre difieren hay también esta identidad. En cuanto a la identidad por el accidente es de toda 
claridad que no se trata de una identidad esencial, pues dos cosas se dicen de esta manera idénticas sólo porque coinciden en la misma substancia individual (Met. V, 6, 1015b 22 y 9, 1017b 27), pero ello no viene en absoluto exigido por la esencia. Es dudoso si la identidad por la propiedad lo es esencialmente o no. Tiendo a pensar que sí, aunque este asunto lo dejaremos aquí de lado.

Nos hallamos en posesión de esta lista, más o menos completa, de las maneras en que puede analizarse una proposición simple desde el punto de vista de los predicables. También tenemos un procedimiento para representarlas simbólicamente. Estamos, pues, en condiciones de examinar lo que he llamado lógica de los predicables, que no trata sino de las leyes de consecuencia válida que tienen su fundamento en este modo de analizar las proposiciones. Para ello, consultaremos algunos de los muchos textos en que Aristóteles emplea este recurso metodológico - sin duda uno de los más frecuentes, al menos en sus primeras obras.

Empezaremos por algunos textos que atañen a razonamientos con estructura de la primera figura silogística, o sea, a razonamientos con dos premisas y tres términos, uno de los cuales se repite en las premisas, una vez como sujeto y otra como predicado.

III. "Es necesario que las definiciones de los géneros se prediquen de la especie y de las cosas que participan de la especie" (Top. IV, 2, 122b 69).

Sobre este texto deben hacerse las aclaraciones siguientes:

a) Por "definición" ( $\lambda$ ó $\gamma \circ \varsigma$ ) podemos entender la composición de género y diferencia, o cualquiera de estas cosas por separado, pues "toda diferencia específica, juntó con el género, produce una especie"(Top. VI, 6, 143b 8).

b) La especie puede ser infima o intermedia (lo que es género respecto de sus inferiores pero especie respecto de los conceptos superiores). 
c) Lo que participa de la especie ínfima son sólo los individuos, mientras que de la especie intermedia, o género no supremo, participan las especies inferiores y sus individuos (Top. IV, 2, 122b21).

Una vez hechas estas aclaraciones, pòdemos ver cómo en esta breve frase se contiene implícitamente la ley siguiente:

1) $B^{g} A \wedge A^{B} C \rightarrow B^{(g)} C$

Que viene a decir que el género $B$ (una parte del enunciado) de un género $A$ se dice de una especie $C$ de éste último. También expresa nuestro texto, entre otras, estas leyes:

2) $\mathrm{B}^{\mathrm{g}} \mathrm{A} \wedge \mathrm{A}^{\mathrm{e}} \mathrm{a} \rightarrow \mathrm{B}^{(\mathrm{g})} \mathrm{a}$

3) $(\mathrm{BC})={ }^{\mathrm{de}} \mathrm{A} \wedge \mathrm{A}^{\mathrm{g} a} \rightarrow(\mathrm{BC})^{(\mathrm{g})} \mathrm{a}$

Sin embargo cuando, en un razonamiento de esta misma estructura, la primera premisa es la predicación de un accidente, no vale la conclusión, como se colige del párrafo siguiente:

IV. "(...) por lo que respecta a las cosas que están en un sujeto [los accidentes] en la mayor parte de los casos ni el nombre ni la definición se predican del sujeto; pero a veces nada impide que el nombre se predique del sujeto, aunque sea imposible que se predique la definición, por ejemplo, blanco, que está en un sujeto, a saber, en el cuerpo, se predica de un sujeto (puesto que el cuerpo se dice que es blanco); sin embargo, la definición de blanco jamás se predica del cuerpo" (Cat. 5, 2a 28).

Dejando de lado el caso de los accidentes que no se predican del sujeto $^{20}$, sólo nos fijaremos en aquéllos que permiten una

${ }^{20}$ De un sujeto no se predica el nombre de un accidente cuando ha de darse una inflexión a dicho nombre para que pueda decirse del sujeto (es decir cuando lo que puede predicarse es un parónimo). Asi "valentía", que es el nombre de un accidente 
predicación, dado que esto es lo que ahora nos interesa. Lo que el texto IV dice respecto de estos accidentes es que su definición y sus partes - género y diferencia- no se pueden decir del sujeto. No son, pues, válidas las fórmulas siguientes ${ }^{21}$.

*4) $\mathrm{C}^{\mathrm{B}} \mathrm{B} \wedge \mathrm{B}^{\mathrm{a} a} \rightarrow \mathrm{Ca}$

*5) $(\mathrm{CD})={ }^{\text {de }} \mathrm{B} \wedge \mathrm{B}^{\mathrm{a}} \mathrm{a} \rightarrow(\mathrm{CD}) \mathrm{a}$

*6) $\mathrm{C}^{\mathrm{di}} \mathrm{B} \wedge \mathrm{B}^{\mathrm{a}} \mathrm{a} \rightarrow \mathrm{Ca}^{22}$

No se puede, en efecto, concluir que la definición de lo dicho accidentalmente o cualquiera de sus partes (género y diferencia) se digan necesariamente del sujeto del accidente. $Y$ así, por tener la estructura de la fórmula 4 , no es válido este rạzonamiento que sirve a Aristóteles de ejemplo en el texto citado:

C) Blanco es color

Este cuerpo es blanco

Luego, este cuerpo es color.

\section{Los sofismas a la luz de la lógica de los predicables}

a) El primer tipo de sofismas del accidente

Veamos ahora la versión aristotélica del intercambio de idénticos, caracterizada porque emplea la noción de "numéricamente idéntico", en vez de idéntico a secas, y porque se aplica sólo sobre sujetos o predicados conforme a la concepción aristotélica de la proposición.

del alma no se predica de ella, pero si su parónimo "valiente" (Cat. la 13). El nombre de estos accidentes, según entiendo, sólo puede decirse de sus inferiores en la categoria correspondiente. En cambio "blanco", que es el nombre de un accidente del cuerpo sí se predica del cuerpo en el que está este accidente.

21 Las fórmulas no válidas llevan un número precedido de un asterisco.

22 Por lo mismo no son tampoco válidas las fórmulas que se hallan al substituir el signo de cosas singulares " $a$ " por un signo de universales como " $A$ ". 
V. "Todo lo que se predica de una [de dos cosas numéricamente idénticas] es necesario que se predique también de la otra, y, de aquello de los que se predica una, es necesario que se predique la otra" (Top. VII, 1, 152b 27, Cfr. Top. VII, $1,15.2 \mathrm{a} 32$ ).

La primera parte de esta regla tiene, sin embargo, una importante restricción que no viene expresada en esta formulación, sino en las Refutaciones Sofisticas, precisamente a la hora de tratar del sofisma del accidente. Esa restricción invalida la regla del intercambio para predicados accidentales que se dicen de cosas numéricamente idénticas por accidente. Cuando se dan estas condiciones, no es necesario que el predicado verdadero de una de las cosas idénticas se diga de la otra.

Esta restricción declara inválidas fórmulas como:

*7) $\mathrm{C}^{\mathrm{a}}{ }_{\mathrm{i}} \mathrm{A} \wedge \mathrm{i} \mathrm{A}={ }^{\mathrm{a} b} \rightarrow \mathrm{Cb}$,

lo cual no impide que esta otra fórmula sea válida:

8) $\mathrm{C}^{\mathrm{a}} \mathrm{A} \wedge \mathrm{A}={ }^{\mathrm{n}} \mathrm{B} \rightarrow \mathrm{CB}$,

pues la identidad que en ellos aparece no es accidental sino esencial $^{23}$.

Pues bien, si simbolizamos el sofisma de Corisco resulta precisamente la fórmula 7 ("El que viene es un desconocido para ti" es una predicación accidental: $C{ }^{a} i A$; "Corisco es el que viene" es una identidad por accidente: $; A={ }^{a} b$ ). Así se pone de manifiesto qué le falta a este sofisma para convertirse en un razonamiento correcto: si comparamos la fórmula 7, que simboliza el sofisma, con, por ejemplo, la fórmula válida 8 , veremos que, si en lugar de una

23 Esta restricción, en realidad, afecta a la regla de intercambio no sólo cuando al predicación es accidental, sino también cuando es de cualquier otro tipo. No valen por ejemplo fórmulas como: ${ }^{*} A^{8}{ }_{i} B \wedge i B={ }^{a}{ }_{i} C \rightarrow A_{i} C$, aunque de esto no vamos a ocuparnos ahora, dado que ninguno de los sofismas del accidente parece responder a fórmulas de este tipo. 
identidad por el accidente, hubiera habido en 7 una identidad por el nombre, tendríamos una fórmula válida. En otras palabras, el sofisma del accidente parece seguir la regla de intercambio, pero no atiende a la restricción que hemos visto. Si ésta explicación fuera la de Aristóteles habríamos descubierto la causa de la apariencia y la causa del error que, a sus ojos, se comete en esta falacia. Antes de continuar debemos pues detenernos para mostrar que los obscuros textos de Aristóteles se entienden perfectamente a la luz de esta interpretación.

En el texto I, Aristóteles señala la causa de la apariencia que provoca los sofismas del accidente: hay, dice al final del texto, unos predicados que tienen la característica de que cuanto se predica accidentalmente de ellos se dice de los sujetos de los que ellos se predican. Esos predicados, conforme a lo que sugiere el principio del texto, son los que no sólo se dicen de sus sujetos, sino que además son idénticos a ellos. Por eso parece que Aristóteles dice aquí que la incapacidad de discernir lo idéntico de lo diverso y la incapacidad de discernir los predicados que transfieren a sus sujetos sus accidentes, constituyen el mismo error.

En este texto no se explicita, sin embargo, a qué tipo de identidad o de predicación se refiere Aristóteles. Para responder a esto hemos de consultar el párrafo II, donde Aristóteles expresa la regla de intercambio de idénticos en lo que a los sujetos (no a los predicados) se refiere, pero añadiendo una restricción que no se halla en el texto I. Este texto contiene dos frases que han de entenderse una por relación a la otra. La primera de ellas, a pesar de su parecido, no ha de confundirse con la que se halla al final del texto I: en esta se dice que no todos los accidentes de un predicado se dicen de su sujeto, mientras que, en el texto II 'se dice que no' es necesario que los predicados de un accidente se digan de los sujetos del accidente. Con ello expresa Aristóteles una regla negativa que ya hemos visto (vid. supra texto IV), y que prohibe concluir que los predicados esenciales o accidentales de un accidente se digan necesariamente de aquello de lo que el accidente se dice. 
Ahora bien, por "accidente", en el texto II, debe entenderse no sólo los universales que se predican accidentalmente, sino también los accidentes exclusivos o usados como propiedad, de que arriba hemos hablado. Incluso Aristóteles, a la luz de la segunda frase del texto II, parece que se refiere especialmente a los accidentes exclusivos. En efecto, en esa segunda cláusula Aristóteles expresa la regla de intercambio de idénticos, pero declara que esa regla sólo es válida cuando esos sujetos son numéricamente idénticos ("son una y la misma cosa") por la esencia ("indiferenciados por la esencia"). Con ello lo que directamente se rechaza es que dicha regla sea válida cuando hay identidad numérica no esencial o accidental, es decir, cuando se dice un accidente exclusivo de un sujeto singular. Por tanto, como la segunda frase pretende aclarar la primera, a lo que principalmente se refiere Aristóteles en ésta es al accidente exclusivo.

A la luz, pues, de este segundo texto se entiende lo que Aristóteles dice en el texto I: para que un accidente que se dice de un término de una proposición, se diga necesariamente del otro ha de haber identidad por la esencia o predicación entre términos esencialmente idénticos y no una identidad accidental o cualquier otro tipo de predicación. De esta manera, a mi juicio, queda plenamente fundamentada en las palabras de Aristóteles la restricción de la regla de intercambio.

Falta por aclarar, sin embargo, por qué señala Aristóteles, en el texto I, la necesidad de distinguir lo idéntico de lo que no lo es, en vez de señalar, como sería de esperar, la necesidad de distinguir entre lo idéntico por la esencia y lo idéntico por accidente. La explicación es, a mi juicio, bien sencilla: Lo accidentalmente idéntico lo es sólo de manera secundaria o parcial, pues se da unidad en la materia y no en la forma. En cambio la identidad esencial lo es de manera plena, con unidad de materia y de forma. Asi se explíca que Aristóteles, un poco después del párrafo II diga que Corisco y el que se acerca no son lo mismo (Ref. Sof. 24, 179b 2) y que, sin embargo, en los Tópicos diga que hay identidad por el accidente entre Sócrates y un accidente exclusivo como "el que está sentado" (Top. I, 7, 103a 32 ss.). 
La restricción expuesta del principio de intercambio permite a Aristóteles resolver también una "aporia lógica" que se le presenta en la Física a propósito del movimiento ${ }^{24}$. A juicio de Aristóteles, el móvimiento por el que un motor mueve y por el que un móvil es movido es un solo acto y no dos (el acto del motor y el acto del móvil) (Fis. III, 3, 202a 17 y 202b 8). Al haber, pues, un solo acto, serán idénticos el acto del motor y el del móvil. Mas si los actos son idénticos y de uno de ellos puede decirse que está en el motor, también, por intercambio, lo estará el otro, de manera que en el motor de dará a la vez que mueve y es movido. Así, por ejemplo, si la enseñanza es un solo acto, tanto del que enseña como del que aprende, este acto concreto de aprender será idéntico a este otro acto concreto de enseñar. Mas como este último está en el que enseña, también, por intercambio, lo estará el otro, de modo que, en virtud de un solo acto, a la vez estará en el que enseña el enseñar y el aprender, lo cual es absurdo (Fis. III, 202a 36 ss.). El razonamiento que aquí se efectúa es el siguiente:

D) Este acto de enseñar está en el que enseña

Este acto de enseñar = este acto de aprender

Luego, este acto de aprender está en el que enseña

La respuesta de Aristóteles es la misma que ofrece para el sofisma de Corisco: la identidad que aparece en la segunda premisa no es esencial sino accidental y, por tanto, no cabe aplicar la regla de intercambio. Que esta es la respuesta de Aristóteles se colige, a mi juicio, del siguiente texto:

VI. "No es necesario que el que enseña reciba la enseñanza $\mathrm{y}$, si bien la acción y la pasión son una misma cosa, no lo son sin embargo en el sentido de que su definición - la que expresa la esencia- sea una, como ocurre entre abrigo y gabán, sino como la carretera de Tebas a Atenas es la misma que la de Atenas a Tebas, como ya se ha dicho más arriba;

24 Cfr. M. Mignuccl: "Puzzles about identity. Aristotle and his greek commentators", en Aristoteles Werk und Wirkung, Berlin: W. de Gruyter 1985, p. 66 ss. 
pues todas las mismas cosas no pertenecen a los que son idénticos de cualquier manera, sino a los que son idénticos en el ser (Fis. III, 202b 10-16).

Aunque entre este aprendizaje y esta enseñanza hay identidad numérica (son una y la misma cosa), no hay por ello identidad esencial, a diferencia de lo que ocurre entre abrigo y gabán, que es el ejemplo preferido por Aristóteles de identidad por el nombre (una de las identidades numérica por la esencia). Esta es, según el texto citado, la razón por la que no puede aplicarse aquí el intercambio de idénticos, pues tal regla no es válida para cosas que son idénticas "de cualquier manera", sino sólo para las cosas que son idénticas "en el ser" o, lo que es igual, para las cosas idénticas por la definición o esencialmente idénticas. En otras palabras, puesto que la única identidad numérica no esencial es la identidad por el accidente, la razón por la que no es válido el razonamiento que nos ocupa es que la identidad de la segunda premisa es accidental. A mi juicio, este texto y los que arriba extrajimos de las Refutaciones Sofisticas contienen una misma doctrina. La única diferencia es que, en esta última obra, Aristóteles parece colocar la identidad por el accidente fuera de la identidad, mientras que en la Física, más acorde con la subdivisión de la identidad que aparece en los Tópicos, la incluye en la identidad numérica, pero destacando que no lo es por la esencia. Lo mismo viene a decir Aristóteles líneas después, pues afirma que la enseñanza y el aprendizaje no son lo mismo en el sentido principal de la palabra (xv@íos), aunque son idénticos porque pertenecen o designan el mismo sujeto singular: el movimiento.

VII. "En general decimos, pues, que ni la enseñanza y el aprendizaje, ni la acción y la pasión, son lo mismo en

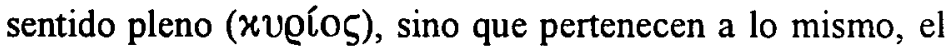
movimiento: en efecto, el acto de esto en aquello y el acto de aquello bajo la acción de esto son diferentes en cuanto a la definición" (Fis. III, 3, 202b 19-22).

Casi todos los textos de la Refutaciones Sofisticas que hablan del sofisma del accidente se acomodan muy bien a la interpretación que he presentado. Debo reconocer, sin embargo, que hay un párrafo que 
parece escapar a ella. Me refiero al texto donde Aristọteles presenta por primera vez este paralogismo. Dice así:

VIII. "Los paralogismos debidos al accidente se dan cuando se piensa que una cosa cualquiera pertenece de igual manera a la cosa y a su accidente. Pues, en efecto, como en la misma cosa se dan accidentalmente muchas, no es necesario que todas ellas pertenezcan a todos los predicados y a aquello de que se predican. Así si Corisco es distinto de hombre, es distinto de sí mismo, pues es hombre. O si es distinto de Sócrates y Sócrates es hombre, dicen que ha de admitirse que es distinto de hombre, porque se da accidentalmente que, aquél que se dijo que es distinto, es hombre" (Ref. Sof. 4, 166b 28-36).

La primera frase de este párrafo es similar a la primera del texto II y no ofrece por tanto dificultad. La dificultad se halla en el resto y muy especialmente en los ejemplos que aquí ofrece Aristóteles. Si por el momento dejamos estos de lado y nos ocupamos de la doctrina, la única interpretación que se me ocurre es que Aristóteles se está fijando en el fundamento ontológico por el que son incorrectos los sofismas del accidente.

En la Fisica dice Aristóteles que ha de distinguirse entre los acontecimientos que suceden en virtud de una causa necesaria o por si y los que suceden por una causa azarosa o accidental. Asi que el que posee el arte de la construcción produzca una casa es causa necesaria, pero si alguien, además de ser constructor es músico, se puede decir que el músico ha producido una casa, aunque esto no ocurra por una causa necesaria, sino por una causa indeterminada o azarosa. Una de las razones que Aristóteles ofrece para explicar que las causas accidentales sean fortuitas o azarosas ès que los accidentes de una misma cosa son infinitos:

IX. "Ahora bien, cuando tales hechos se producen por accidente, decimos que son efectos de la fortuna: en efecto, de la misma manera que al ser se aplica la distinción de lo que es por sí y por accidente, asi puede aplicarse a la causa: 
por ejemplo, el arte de construir es la causa por sí de la casa, lo blanco y lo músico lo son por accidente. La causa por sí es determinada, la causa accidental es indefinida, pues la multitud de los accidentes posibles de una cosa es infinita" (Fis..II, 5, 196b 23-29).

Al principio de este párrafo Aristóteles establece una analogía entre los seres, de una parte, y las causas del cambio, de otra: de la misma manera que se distinguen los seres por sí de los seres por accidente, se distinguen los cambios que se producen por causas por si de los que se producen por causas accidentales. Pues bien, en el párrafo VIII, al decir que en una cosa se dan accidentalmente muchas, quizás Aristóteles extiende a los seres accidentales la explicación que da en el texto IX de las causas accidentales. La infinidad de los accidentes es razón, en el movimiento, de que los accidentes sean causas azarosas y, en los seres, es razón de que no necesariamente haya fundamento para el nexo predicativo entre los accidentes. La segunda frase del párrafo VIII vendria, pues, a decir que, como los accidentes de la cosa que oficia de término medio en nuestro sofisma, son muchos, es indeterminado y no necesario que estos accidentes puedan decirse unos de otros. Asi, en el sofisma de Corisco, tendríamos que, puesto que ser el que viene y ser conocido por. ti son accidentes de la misma cosa - Corisco-, no necesariamente se sigue que se pueda decir que el que viene es un desconocido para ti.

El comentario que al final del párrafo VIII hace Aristóteles a su segundo ejemplo, abona esta conjetura, pues parece que atribuye el error del sofisma al hecho de que en la cosa (Sócrates) que oficia de término medio se da accidentalmente ser distinto de Corisco y ser hombre. Sin embargo, esta conjetura topa con la dificultad de los ejemplo mismos que Aristóteles elige, pues en ellos no parece que se den predicaciones accidentales. Si atendemos a este párrafo y nos dejamos guiar por sus ejemplos, nada tiene de extraño que muchos autores hayan creído que Aristóteles usa aquí el término accidente en el sentido amplio de predicación. 
Con todo, creo que la interpretación ontológica ofrecida, aún necesitada como está de muchas precisiones, es cuando menos plausible y que, quizás, las dificultades que presentan los ejemplos son meras deficiencias en la elección de los mismos.

b) El sofisma del consecuente

Otra regla de la lógica de los predicables es lo que podríamos llamar la transitividad de la identidad: si dos cosas son idénticas a una tercera, con cualquier género de identidad numérica, son idénticas entre sí (Top. VII, 1, 152a 31 y Ref. Sof. 6, 168b 32) ${ }^{25}$. Son, pues, válidas entre otras las fórmulas siguientes:

8) $\mathrm{A}={ }^{\mathrm{n}} \mathrm{B} \wedge \mathrm{C}={ }^{\mathrm{n}} \mathrm{B} \rightarrow \mathrm{A}={ }^{\mathrm{n}} \mathrm{C}$

9) $\mathrm{a}={ }^{\mathrm{a}} \mathrm{i} \wedge \wedge{ }_{i} \mathrm{C}={ }^{\mathrm{a}} \mathrm{iB} \rightarrow \mathrm{a}={ }^{\mathrm{a}} \mathrm{i} \mathrm{C}$

Los sofismas que Aristóteles llama del consecuente se producen cuando creemos que dos cosas son la misma porque poseen accidentes sensibles iguales, como ocurre en este repugnante ejemplo:

E) La miel es amarillenta

La bilis es amarillenta

Luego, la bilis es miel

(Ref. Sof. 5, 167b 5)

Pues bien, estos paralogismos son argumentaciones que parecen cumplir la ley de transitividad de idénticos, pues, tal como la presenta Aristóteles, esta ley tiene, como los sofismas del consecuente, la estructura de la segunda figura, es decir, el término medio ocupa por dos veces el lugar del predicado. Sin embargo, a pesar de la apariencia, no cumplen tal ley, pues en lugar de ser identidades, las premisas sólo constan de predicaciones accidentales

25 La restricción a la regla de intercambio de idénticos no invalida la transitividad de la identidad, pues dicha restricción sólo afecta a los razonamientos donde hay una predicación accidental sin identidad. 
sin identidad. Tales son pues, a juicio de Aristóteles, las causas de la apariencia y del defecto de estos paralogismos, lo cual se ve claramente en este texto:

$\mathrm{X}$. "Los [paralogismos] debidos al consecuente son una parte del accidente, pues el consecuente es algo que sobreviene accidentalmente. Pero difiere del accidente en que el accidente sólo es posible tomarlo sobre una cosa. Por ejemplo: que es lo mismo lo amarillento y la miel y lo blanco y el cisne; en cambio el consecuente se da siempre en varias cosas; en efecto, las cosas que son idénticas a una tercera cosa pensamos que son también idénticas entre sí: . por eso surge una refutación en virtud del consecuente. Pero esto no siempre es verdad, como ocurre si es por accidente; en efecto tanto la nieve como el cisne son idénticos a lo blanco" (Ref. Sof. 6, 168b 27-35).

Aquí Aristóteles señala la transitividad de los idénticos como la ley que parecen seguir estos razonamientos, $y$, tras ello, dice que no siempre vale esta ley "como ocurre si es por accidente". Lo que con esto quiere decir Aristóteles no es que para la identidad accidental no valga la regla de transitivisdad. Más bien, creo yo, quiere decir que cuando de dos cosas se predica lo mismo accidentalmente, no se cumplen las condiciones de la regla de transitividad, por falta de identidad, pues en la predicación accidental no se involucra necesariamente la identidad entre sus términos. Efectivamente, en un párrafo anterior Aristóteles señala que el error que se comete en el sofisma del consecuente consiste en creer que el consecuente (una clase de accidente) es convertible en la predicación, lo cual es una caracteristica exclusiva de los predicados que son idénticos numéricamente al sujeto (Cfr. Top. I, 8, 103b l ss. y. I, 7. 103a 25 ss.). He aquí el texto de Aristóteles:

XI. "La refutación debida al consecuente se da por creer que la consecuencia es reversible, en efecto, cuando, al existir esto, necesariamente existe aquello, también, creen 
algunos, al existir lo segundo existirá necesariamente lo primero" (Ref. Sof. 5, 167b 1-3) ${ }^{26}$.

c) El segundo tipo de sofisma de accidente

A la luz de la lógica de los predicables, hemos podido ya resolver uno de los sofismas del accidente y los sofismas del consecuente. Ahora debemos examinar el sofisma del perro progenitor para ver si se explica de igual manera.

Para este fin hemos de recurrir a un texto del Peri hermeneias, en el cual Aristóteles se da a la tarea de exponer cuándo se pueden componer y descomponer los predicados dichos de un mismo sujeto.

En esta obra ofrece Aristóteles unos ejemplos de razonamientos sofisticos que tienen la misma estructura que el del perro.

F) Este hombre es bueno

Este hombre es zapatero

Luego, este hombre es buen zapatero

(De Int. 11, 20b 35)

Es uno de dichos ejemplos. La dificultad que se nos presenta es que hay razonamientos que parecen tener esta misma estructura y que, sin embargo, son perfectamente correctos. Asi:

G) Juan es hombre

Juan es blanco

Luego, Juan es hombre blanco

H) Juan es animal

26 Queda por aclarar el parentesco que Aristóteles encuentra, en el párrafo X y en otros lugares (Ref. Sof. 7, 169b 5), entre el sofisma del accidente y el del consecuente. Parece que lo que Aristóteles quiere decir al afirmar que éste es una parte de aquél, es que el consecuente afirma que un accidente que previamente se habia dicho de una cosa se da también en otra. Tal es, a mi juicio, lo que significa lá atirmación según la cual el accidente se da sobre una cosa, mientras que el consecuente se da en dos. 
Juan es racional

Luego, Juan es animal racional

La razón por la que el primero es un sofisma mientras que estos otros son razonamientos consecuentes, se puede entender gracias a una regla que aparece de alguna manera en los mencionados textos, y en especial de la primera parte del Peri hermeneias.

Esa regla viene a decir:

XII. Cuando dos predicados se dicen de un sujeto individual, se puede predicar la combinación de ambos predicados, a no ser que: a) estos predicados sè digan uno del otro esencialmente, y a no ser que: b) ambos predicados se digan como accidentes del citado sujeto ${ }^{27}$.

Por tanto, si se cumplen las dos restricciones señaladas en la regla XII, se pueden decir compuestos dos predicados que previamente se han dicho por separado de un sujeto. Asi es válida la fórmula:

12) $A^{c} b \wedge B^{a} b \rightarrow(A B) b$,

que simboliza el razonamiento $G$, y también

13) $A^{g} b \wedge B^{d i} b \rightarrow(A B) b$

que simboliza el razonamiento $\mathrm{H}$.

Pero estas combinaciones de predicados no pueden hacerse si los predicados son accidentales, pues en tal caso se pueden cometer sofismas como el del perro padre o el del buen zapatero, que se simbolizan por medio de la fórmula:

27 "Es pues evidente que si alguien establece sin más que los compuestos tienen lugar, resulta que dice muchos absurdos. Digamos ahora cómo se ha de disponer. De las cosas predicadas y de las que resultan sujetos de predicación, cuantas se dicen accidentalmente bien de la misma cosa o bien de otra, no serán unitarias (...) Además tampoco lo serán cuantas cosas estén contenidas la una en la otra" (De Int. 11, 21a 5-16). 
*14) $A^{a} b \wedge B^{a} b \rightarrow(A B) b$,

que es inválida, por la restricción $b$ de la regla.

\section{d. La redundancia}

El arte de los sofistas perseguía el desprestigio del contrincante ante el auditorio por cualquier medio. Uno de ellos es la refutación sofistica, que consiste en llevar al interlocutor, por medio de un razonamiento incorrecto, a concluir una proposición que contradice otra previamente aceptada. Este es el fin de razonamientos como el de Corisco, que permite establecer que no conoces a Corisco cuando previamente has aceptado que Corisco es tu amigo.

Pero el desprestigio del interlocutor puede alcanzarse haciendo aceptar al interlocutor no una proposición contradictoria, sino una falsedad manifiesta o incluso una proposición mal construida (Ref. .Sof. 4). Tal es el caso de las proposiciones redundantes, donde un mismo predicado es dicho varias veces de un mismo sujeto. El procedimiento que para esto se sigue consiste en hacer que el contrincante acepte una composición de predicados de tal manera que uno esté contenido en el otro, como ocurre entre el género, la diferencia o la propiedad por una lado y la especie por otro ${ }^{28}$. Así ocurre con el siguiente razonamiento:

I) Esto es una nariz

Esto es chato

Luego, esto es una nariz chata

28 Aristóteles habla en varias ocasiones de la nugatio o redundancia (Ref. Sof. 13 y 31; Top. V, 2, 130a 39 y VI, 3, 140b 27). Estos sofismas se construyen, si no me - equivoco, componiendo dos términos universales, uno de los cuales contiene al otro en su intensión. Asi ocurre con los términos relativos que contiene implícitamente su correlativo (v.g.: doble es doble de la mitad). Lo mismo ocurre con las propiedades, en cuya definición entra la especie de que es propiedad y con la especie, en cuya definición entran género y diferencia. Sólo estos dos últimos casos se resuelven con la ayuda de la lógica de los predicables. 
El que haya aceptado este razonamiento ya está perdido, pues basta con señalar que chato se define como nariz cóncava para, por substitución de los idénticos, obtener desde la conclusión la proposición:

J) Esto es una nariz cóncava (Ref. Sof. 13, 173a 34 y 173b 10),

lo cual es para Aristóteles una proposición redundante, absurda y obscura (Top. V, 2, 130a 33).

$\mathrm{El}$ error, en este caso, procede de no haber atendido a la primera restricción de la resolución de predicados. En efecto, conforme a una ley que no hemos visto aquí ${ }^{29}$, tenemos:

15) $A^{\mathrm{g}} \mathrm{a} \wedge \mathrm{B}^{\mathrm{e}} \mathrm{a} \rightarrow \mathrm{A}^{\mathrm{B} B}$,

donde el antecedente simboliza las premisas del razonamiento de la nariz. Ahora bien, la primera restricción de la composición de predicados prohíbe que se compongan aquellos que se dicen entre sí esencialmente (o que están contenidos el uno en el otro), lo cual se expresa por medio de las fórmulas siguientes:

*16) $\mathrm{B}^{\mathrm{di}} \mathrm{A} \rightarrow(\mathrm{BA}) \mathrm{a}$

$\left.{ }^{*} 17\right) \mathrm{B}^{\mathrm{P}} \mathrm{A} \rightarrow(\mathrm{BA}) \mathrm{a}$

*18) $\mathrm{B}^{\mathrm{B}} \mathrm{A} \rightarrow(\mathrm{BA}) \mathrm{a}$

entre otras. Por consiguiente, no cabe afirmar a partir de las premisas del razonamiento de la nariz, la conclusión según la cual Corisco es hombre bipedo.

\section{e. La falacia "secundum quid ad simpliciter"}

29 Esta ley, evidente por otra parte, puede colegirse de la afirmación aristotélica según la cual la definición y sus partes (género y diferencia) son del universal y no de los singulares (Cfr. J. M, GAMBRA: "La lógica aristotélica de los predicables", Anuario Filosófico XXI/2 (1988), p. 101). 
Finalmente hay sofismas que se deben a una resolución o descomposición incorrecta de predicados. Así ocurre con estas argumentaciones:

K) Este es buen zapatero

Luego, éste es bueno

L) Esto es un hombre muerto

Luego, esto es un hombre

Se comete una de las falacias de lo dicho bajo un aspecto a lo dicho sin más (secundum quid ad simpliciter) $)^{30}$.

La regla que rige la descomposición de predicados aparece al final del capitulo 11 del Peri hermeneias, y puede expresarse de la manera siguiente:

XIII. "Se puede concluir la predicación simple desde una predicación compuesta de dos predicados con tal de que: a) no haya contrariedad entre dichos predicados y b) uno de ellos no sea un accidente del sujeto",31.

Asi, por la primera restricción, no es correcta la segunda argumentación, que puede simbolizarse por medio de la fórmula inválida siguiente:

*19) (AB)a $\wedge \mathrm{A \# B \rightarrow Aa,}$

30 "Los paralogismos debidos a que tal cosa se diga de manera absoluta o bajo algún. aspecto y no propiamente, se dan cuando lo que se enuncia como particular se toma como lo dicho de manera absoluta" (Ref. Sof. 5, 166b 32. Cfr. Ref. Sof. 6, 168b 11; 7, 169 b 10; 25, 180a 23; Ret. II, 24, 1402a 3).

31 "Es verdadero hablar de una cosa determinada incluso simpliciter (...) Pero no siempre, sino que, cuando en lo que se añade se contiene un opuesto del que se sigue una contradicción no es verdadero, sino falso (...) Así, de cuantas predicaciones que no contengan nunguna contradicción, si se substituyen los nombres por las definiciones y se predican por sí y no por accidente, en estos casos será verdadero hablar de la cosa determinada incluso simpliciter" (De Int. 11, 21 a 18-32; cfr. sobre esta regla J. M. GAMBRA: "La regla aristotélica de resolución de predicados", Philosophica Malacitana 2 (1989), p. 73-86). 
donde el signo \# expresa la relación de contrariedad entre términos.

Tampoco vale, por la segunda restricción el sofisma del buen zapatero, que viene a simbolizarse por medio de la fórmula no válida:

*20) (AB)a $\wedge \mathrm{A}^{\mathrm{a} a} \rightarrow \mathrm{Ba}$

La razón última de estas restricciones, tanto de la resolución como de la composición de predicados, que impiden realizar estas operaciones cuando los predicados son accidentales se halla, a mi juicio, en la frase siguiente:

XIV. "Sólo en el caso de los accidentes cabe que algo se diga bajo algún aspecto y no universalmente" (Top. II, 1, 109a 13).

\section{Conclusión}

La lógica de los predicables, de la cual sólo he presentado aquí sus grandes rasgos, constituye quizás la primera silogística en que Aristóteles pensó. En efecto, Aristóteles define el razonamiento o silogismo de manera exactamente igual en sus primeras obras (Top. I, 1, 100a 25), antes del descubrimiento del silogismo cuantificado, y en los Primeros Analíticos (I, 1, 24b 18), donde expone la teoría de estos últimos silogismos. Parece, pues, natural que Aristóteles tuviera una teoría formal del razonamiento previa a la silogística cuantificada. Dicha teoría es, a mi juicio, la lógica de los predicables. Entiendo por tanto errada la apreciación de H. G. Gelber, según la cual el uso de los modos de predicación es una intromisión de la materia en la forma del razonamiento ${ }^{32}$. Igualmente errada es la

32 H. G. GELBER, refiriéndose a la interpretación, perfectamente acorde con lo que he llamado lógica de los predicables, que propone Boecio del cuarto antepredicamento (Cat. 3, lb 10), dice que "subordina el poder de la forma lógica a la naturaleza de los casos a que se aplica. La materia o contenido del argumento tiene en última instancia prioridad sobre la forma de la argumentación a la hora de determinar la validez de la inferencia, pues el contenido determina la naturaleza de la predicación involucrada, de modo que sea o no esencial" ("Fallacy of Accident 
opinión de G. A. Bueno, según la cual en las obras del Organon escritas antes del "gran sistema de lógica silogística (...) los argumentos son tratados de manera informal" y "no hay uso de variables lógicas o un análisis formal del silogismo" ("Aristotle, the Fallacy of Accident and the Nature of Predication", Journal of the History of Philosophy 26 (1988), p. 8). Lo que ocurre, según entiendo, es que Aristóteles emplea como funciones lógicas los modos de predicación y los tipos de identidad numérica en vez de usar la cuantificación como hará después, aunque, falta hace reconocerlo, lo hace de manera harto más desorganizada que lo hará en los Analíticos Primeros.

Es posible incluso que esta lógica sea el paso inmediatamente anterior a la construcción de la silogística cuantificada, cosa que, si no me equivoco sería fácil de justificar con textos de la Analiticos Primeros. Pero eso es otra historia.

También cabe destacar que esta lógica resuelve las paradojas de la denotación y del análisis, por medio de todo un conjunto sistemático de leyes, lo cual es, sin duda, mucho más satisfactorio que las excepciones o soluciones ad hoc a las que han tenido que recurrir los filósofos analíticos.

Sea de ello lo que fuere, es, a mi modo de ver, evidente que muchas partes de las Refutaciones Sofisticas son completamente ininteligibles, sin acudir a esta lógica. Quizás porque los aristotélicos han tendido a olvidar estos modos de razonar y a entender los sofismas desde el silogismo cuantificado, se ha producido el curioso fenómeno de la tergiversación de las refutaciones al que aludi al principio.

and the Dictum de omni: late Medieval Controversy over a Reciprocal Pair", Vivarium XXV-2 (1987), p. 113) 


\section{Apéndice polémico}

\section{Sobre la traducción de $\sigma v \mu \beta \varepsilon \beta \eta x o ̀ \varsigma$}

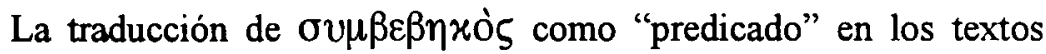
referentes al sofisma del accidente ha tenido numerosos defensores también en nuestros días, como los citados Mignucci y Bueno. A mi juicio, resulta muy dificil mantener esta traducción en la mayoría de los textos. Ya vimos que su base más sólida se halla en el primer texto donde Aristóteles expone el sofisma del accidente. Veamos las principales dificultades con que topa esta interpretación:

1. Cada uno de los paralogismos es denominado por Aristóteles con un término muy bien definido dentro de su vocabulario lógico. Buen ejemplo de ello son la homonimia, la anfibología, la refutación y la petición de principio. También la idea de accidente es definida con gran precisión en los Topicos, cuyo último libro son las Refutaciones, donde se hallan los textos que nos ocupan. Dadas estas circunstancias, sería extraordinario que Aristóteles denominara de manera constante uno de sus sofismas por medio de la palabra "accidente" utilizada, no de manera precisa y clara como lo hiciera antes, sino con el sentido genérico de predicado.

2. En las Refutaciones hay párrafos donde implícita o explíci-

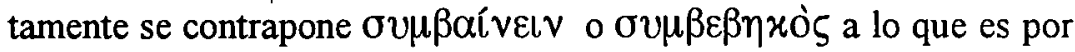
sí, de manera que resulta completamente inadecuado la traducción por predicar o predicado.

a) El caso más claro se halla en Ref. Sof. 8, 170a 4, donde Aristóteles designa el sofisma del accidente por medio de la expresión "el que se debe a poner lo que es por accidente en lugar de

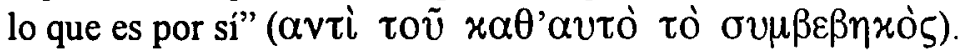

b) En Ref. Sof. 6, 168a 33 ss. Aristóteles trata de mostrar que los paralogismos del accidente no son silogismos correctos y, por ello, al igual que todos lo demás sofismas, se reduce a la ignorancia de la refutación. A este efecto pone el ejemplo siguiente: 
“(...) si el triángulo tiene sus ángulos iguales a dos rectos, $y$ si es por accidente una figura, o un elemento primero, o un punto de partida, no por ser una figura o un punto de partida o un elemento tiene dicho carácter: pues la demostración prueba la propiedad en cuestión, no en cuanto el triángulo es una figura o un elemento primero sino en cuanto triángulo. Y lo mismo en los otros casos. Por tanto si la refutación es una especie de silogismo, un argumento que proceda por accidente no podría ser una refutación" (Ref. Sof. 6, 168a 40$168 \mathrm{~b} \mathrm{5)}$.

Aristóteles dice aquí que la demostración se hace a partir de predicaciones no accidentales. Este mismo asunto lo trata detalladamente en los Analíticos Segundos I, 6, donde contrapone explícitamente los atributos esenciales, necesarios o por sí a los atributos no necesarios o por accidente (An. Post. I, 6, 74b 5-11). En ese mismo capítulo, por ejemplo, dice, afirmando exactamente lo mismo que dice el texto citado, lo siguiente: "Los accidentes, en efecto, no son necesarios, de manera que no se conoce necesariamente una conclusión por su causa, incluso si se usan proposiciones siempre verdaderas si no son por si" (An. Post. I, 6, 75 a 32 ss.).

El pasaje citado explica perfectamente el ejemplo del triángulo: los accidentes de un triángulo no sirven para demostrar sus propiedades necesarias, porque los accidentes, aunque se digan con verdad de un sujeto no permiten conocer la causa. Por consiguiente, si en lo Analiticos Posteriores ha de entenderse que el accidente se opone a los predicados esenciales, también ha de hacerse en las Refutaciones Sofisticas.

c) En el capítulo 24, dedicado a la resolución de los sofismas del accidente, Aristóteles dice, acerca de unos ejemplos similares al del

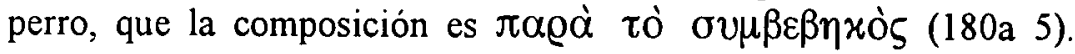
Estos sofismas, como hemos visto, sólo pueden entenderse a la luz del Peri hermeneias 11, 21a 5-18. Pues bien, en ese mismo capitulo, Aristóteles contrapone la predicación por sí a la predicación por accidente, de modo que en las Refutaciones "accidente" también 
debe significar un modo de predicación y no una predicación cualquiera.

El argumento más fuerte que Mignucci aduce en favor de esta traducción consiste en señalar que en el primer ejemplo de sofisma del accidente ofrecido por Aristóteles no hay predicación accidental. Dicho ejemplo reza:

Corisco es diferente de hombre

Corisco es hombre

Luego, Corisco es diferente de sí mismo

(Ref. Sof. 5, 166b 33)

Bien cabe que Aristóteles considerara accidental alguna de las premisas, por ejemplo, la primera. Pero sea de ello lo que fuere, el argumento de Mignucci pierde toda su fuerza si nos percatamos del carácter especial que tienen los ejemplos aristotélicos de sofismas: no están construidos en orden a esclarecer la doctrina de las Refutaciones, sino que están tomados de disputas sofisticas previas (varios de ellos se encuentran en las obras de Platón) y Aristóteles trata de encajarlos en los moldes de su teoría. De ahi su gran interés, pero también la gran dificultad que a veces presentan para ser entendidos a la luz de sus doctrinas, que llega incluso a la inadecuación.

\section{La identidad numérica}

Mignucci, en el artículo citado, rechaza que Aristóteles ponga restricción alguna a la regla de intercambio de idénticos (p. 84). A su juicio, lo que yo entiendo que no es más que una restricción constituiría una contradicción entre el texto de los Tópicos, por un lado, y los párrafos citados de la Física y de las Refutaciones Sofisticas, por el otro (p.70 y 84). Mi postura, en la linea de lo que Mignucci llama interpretación tradicional (p. 69), se reduce a entender que la identidad numérica a la que Aristóteles se refiere en su regla de intercambio (Top. VIII, 1, 152b 27) no incluye la identidad por accidente, lo cual viene expuesto en el párrafo II de las Refutaciones y en la Física III, 3. Mignucci, por el contrario, con el 
fin de que dicha regla "mantenga toda su fuerza de condición necesaria para la identidad" (p. 84) pretende que Aristóteles habla en dicha regla de una sola identidad, la identidad por la esencia, el ser o la definición, y que - $\mathrm{y}$ esto es lo grave- esta identidad es lo mismo que la identidad numérica:

"Lo que aquí se entiende por identidad en la definición o identidad en el ser es algo diferente. A mi juicio significa la identidad en la definición de lo que es denotado por los términos de los que se dice que son idénticos: $x$ e $y$ son idénticos en el ser si lo que es denotado por $x$ tiene la misma definición que lo que es denotado por $y$. Es fácil convencernos de que la identidad en el ser, tomada en este sentido, es simplemente lo mismo que la identidad numérica. Si $x$ e $y$ denotan uno y el mismo objeto, la definición (si la hay) de lo que es denotado por $x$ no puede ser diferente de la definición de lo que es denotado por $y$, puesto que uno y el mismo objeto no puede tener dos definiciones. Por la misma razón, si lo que es denotado por $x$ y lo que es denotado por $y$ tienen la misma definición $x$ e $y$ denotan el mismo objeto" (p. 72).

Verdad es que si dos términos denotan la misma cosa, la definición de lo que denotan es la misma ¿quien podría dudarlo? No tan verdad es que si las cosas denotadas por los nombres tienen la misma definición, entonces son la misma cosa, pues, si bien esto es verdad para los términos universales, no lo es para los singulares: Pedro y Juan se definen de la misma manera, pero no son el mismo, son idénticos por la especie pero no numéricamente idénticos. Pero aunque todo esto fuera indudable, no veo cómo se sigue de ello que la identidad por la definición o en el ser sea lo mismo que la identidad numérica: la identidad numérica se da cuando los nombres son diversos y la cosa es materialmente una. Mignucci señala esto con toda claridad (p. 72), pero inexplicablemente se olvida de que los nombres de cosas idénticas pueden, según Aristóteles, expresar formas diversas. En efecto, como se ha visto, la identidad numérica es de cuatro clases y sólo en dos de ellas, la identidad por el nombre y la definición, el significado de los nombres tiene la misma 
definición. En la identidad por el nombre y la definición, no sólo la cosa significada es una, sino que además los nombres designan la misma forma de esa cosa, que en este caso es su definición. Pero, sin dejar de ser una sola cosa la que los nombres significan, puede que éstos designen formas diferentes de la misma cosa. Así ocurre, como hemos visto, cuando, por ejemplo, se llama algo, de una parte, por su 1 smbre y, de otra, por un accidente que sólo a él conviene. En tal caso tenemos una identidad por el accidente. Sigue siendo verdad que la cosa designada tiene una sola definición, un ser o una esencia única. Pero de ello no se sigue, como pretende Mignucci, que si dos términos son numéricamente idénticos hayan de significar la misma cosa por medio de su definición.

Mignucci parece empeñado en escamotear lo accidental, tanto en la predicación y en la identidad como en el sofisma que lleva este nombre. De esta manera distorsiona el pensamiento lógico de Aristóteles, quizás, porque su intento es entenderlo desde la semántica fregeana, en vez de hacerlo desde la metafisica del propio Aristóteles. 
Copyright of Tópicos. Revista de Filosofía is the property of Universidad Panamericana and its content may not be copied or emailed to multiple sites or posted to a listserv without the copyright holder's express written permission. However, users may print, download, or email articles for individual use. 